First publ. in: Cellular and Molecular Life Sciences 62 (2005), 23, pp. 2727-2738

\title{
Molecular guardians for newborn proteins: ribosome-associated chaperones and their role in protein folding
}

\author{
R. D. Wegrzyn and E. Deuerling* \\ Zentrum für Molekulare Biologie (ZMBH), Universität Heidelberg, Im Neuenheimer Feld 282, \\ 69120 Heidelberg (Germany), Fax: +49 6221 545894, e-mail: e.deuerling@ zmbh.uni-heidelberg.de
}

Received 29 June 2005; received after revision 4 August 2005; accepted 18 August 2005

\begin{abstract}
A central dogma in biology is the conversion of genetic information into active proteins. The biosynthesis of proteins by ribosomes and the subsequent folding of newly made proteins represent the last crucial steps in this process. To guarantee the correct folding of newly made proteins, a complex chaperone network is required in all cells. In concert with ongoing protein biosynthesis, ribosome-associated factors can interact directly with emerging nascent polypeptides to protect them from degradation or aggregation, to promote fold-
\end{abstract}

ing into their native structure, or to otherwise contribute to their folding program. Eukaryotic cells possess two major ribosome-associated systems, an Hsp70/Hsp40based chaperone system and the functionally enigmatic NAC complex, whereas prokaryotes employ the Trigger Factor chaperone. Recent structural insights into Trigger Factor reveal an intricate cradle-like structure that, together with the exit site of the ribosome, forms a protected environment for the folding of newly synthesized proteins.

Key words. Trigger Factor; Ssb; Zuotin; NAC; co-translational protein folding; ribosome.

\section{Introduction}

The biogenesis of proteins by ribosomes is a fundamental and universal process reflecting one of the last steps in the conversion of genetic information into functional proteinaceous units. In living cells several thousand different proteins are synthesized by ribosomes per minute. Immediately upon synthesis all newly produced proteins face two challenges. First, each protein must either find its correct destination in the cytosol or be translocated to another cellular compartment such as the endoplasmic reticulum (ER) or the plasma membrane. Second, the protein has to properly fold into its unique three-dimensional

* Corresponding author. structure to fulfill its biological function. Both processes (protein transport and folding) are initiated co-translationally by ribosome-associated factors that interact with nascent chains to ensure the correct fate of newly synthesized proteins. Targeting factors such as the signal recognition particle (SRP) recognize the signal sequence of secretory proteins and initiate the downstream events that allow for the translocation of a newly synthesized protein. The cellular strategy to promote proper folding of newly synthesized proteins involves a large arsenal of molecular chaperones. These proteins are found in all species and kingdoms and can be divided into two groups based on their cellular localization. Chaperones that bind post-translationally to newly synthesized proteins as soluble components of the cytosol correspond mainly to chaperones of the Hsp70/40 and Hsp60/10 categories. Others 
govern the fate of nascent chains on the ribosome by binding to both the ribosome and the emerging polypeptide, thereby controlling protein folding steps already during protein synthesis. The existence of ribosome-associated chaperones is a highly conserved principle in eukaryotes and prokaryotes, although the parties therein differ completely. Whereas in bacteria the chaperone Trigger Factor (TF) is the midwife for nascent polypeptide chains, protein systems such as the Hsp70-based Ssb machine and NAC (nascent chain-âssociated complex) in eukaryotes are among the first contact partners of nascent proteins at the ribosome. In this review we will focus on the action of ribosome-associated protein factors considered to play a key role in the folding program of newly synthesized proteins, focusing on current knowledge and recent advances regarding these specialized chaperones and their site of action, the ribosome.

\section{The ribosome}

Ribosomes are huge cytosolic machines whose task it is to accurately and efficiently assemble proteins according to genetic instructions. A bacterial cell has around 20,000 ribosomes, whilst a human cell has a few million. Cells that have high rates of protein synthesis, such as liver cells, have a particularly great number of ribosomes. The rate of nascent polypeptide elongation is remarkably high, with about 15-20 amino acids (aa) per second in bacteria and approximately 5-7 aa per second in eukaryotes. Thus an average-sized protein composed of 300 aa is synthesized within 15-60 s under optimal growth conditions in pro- and eukaryotic cells, respectively [1]. Eukaryotic, archaeal and bacterial ribosomes are closely related, despite differences in the number and size of their ribosomal RNA (rRNA) and protein components. The rRNA has the dominant role in translation, determining the overall structure of the ribosome, forming the binding sites for the transfer RNAs (tRNAs), matching the tRNAs to codons in the messenger RNA (mRNA), and providing the peptidyl transferase enzyme activity that links amino acids together during translation. The determination of the complete atomic structures of the large (50S) and small (30S) ribosomal subunits of bacterial and archaeal ribosomes provided new important insights into this advanced macromolecular machine (fig. 1A) [2-4]. The structure of the Haloarcula marismortui large ribosomal subunit exhibits a tunnel of approximately $100 \AA$ in length and an average diameter of $15 \AA$, through which the polypeptide extends while still connected to the peptidyl-transferase center $[5,6]$. The dimensions of the tunnel are large enough to protect a growing polypeptide segment of 30-35 aa in length in an extended conformation from protease digestion [7]. Although a crystal structure of a eukaryotic cytosolic ribosome has not yet been published, a comparative study was performed where the cryo-electron microscopy (EM) structure of the yeast ribosome was used to extrapolate a detailed model of the eukaryotic ribosome using the existing atomic maps of the H. marismortui and Thermus thermophilus ribosomes [8]. Ribosomes of all three kingdoms display a remarkable conservation of the core ribosomal structure.

The polypeptide exit tunnel is largely formed by rRNA, but has significant contributions from two ribosomal proteins. In the archael ribosome, L22 and L4 protrude into the interior of the tunnel where they form, together with the RNA moieties, the narrowest constriction of the exit tunnel (fig. 1A) [5]. In bacteria, the protein L23 similarly extends into the ribosomal tunnel $[9,10]$. Until recently the tunnel was considered to be inert, not interacting with the nascent polypeptide. Recent studies, however, provided evidence that the ribosome can discriminate among certain amino acid sequences as they travel through the tunnel causing a reprogramming of the ribosome during protein biogenesis [11, 12]. In Escherichia coli, a specific sequence motif within the C-terminus of the SecM polypeptide (called arrest sequence, aa 150-166 of SecM: FXXXXWIXXXXGIRAGP) interacts with L22 and rRNA at the narrow restriction site within the ribosomal tunnel to cause translational arrest [11,12]. This elongation arrest is transient and can be overcome by the binding of SecA to the exposed N-terminus of SecM to mediate its subsequent export into the periplasm. Thus, the translational arrest of SecM in E. coli provided the first evidence of the interactive nature of a nascent polypeptide within the ribosomal tunnel with the purpose of regulating ribosomal activity. Since this initial observation, the regulation of ribosomal activity dependent upon internal domains of a nascent chain has also been described in eukaryotes (reviewed in [13]). Notably, the nascent chain sequences described so far which can stall translation of the eukaryotic ribosome bear no sequence homology to the SecM sequence of bacterial cells and are involved in a multitude of different processes such as amino acid metabolism, translocation and mRNA degradation [13, 14].

\section{Assembly line at the ribosomal exit site}

The exit site of the ribosomal tunnel (fig. 1B) is surrounded by rRNA and a repertoire of several ribosomal proteins. Four of these proteins, L22, L24, L23 and L29, are conserved among the three kingdoms of life, whereas the L19, L31 and L39e proteins are not found on prokaryotic ribosomes. This region, where the nascent polypeptide leaves the ribosomal interior and steps into the cytosol, is of crucial importance for the correct positioning of ribosome-associated factors. It was recently discovered that the ribosomal protein L23 serves as 
A

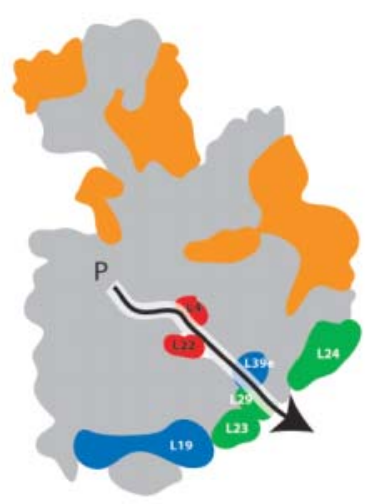

B

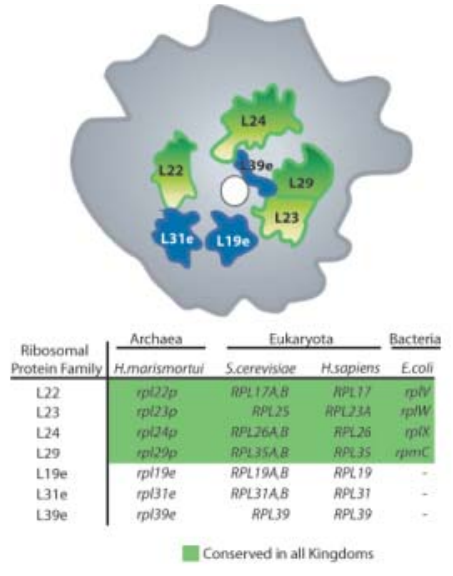

D

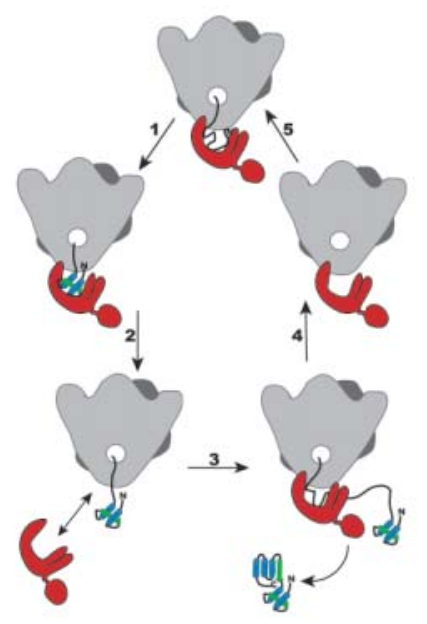

Figure 1. Schematic representation of the large ribosomal subunit, the TF structure and a model of TF's chaperone mechanism. (A) The ribosomal subunit is bisected, showing the interior with the tunnel traversing the subunit indicated by the arrow. The rRNA moieties are given in grey, ribosomal proteins inside the tunnel are highlighted in red, ribosomal proteins at the tunnel exit are shown in green (entirely conserved in evolution) and in blue (found in eukaryotes only), and all other proteins are shown in orange. Drawing based on the structure of Haloarcula marismortui 50S ([2, 5]). (B) Illustration showing the exit site of large ribosomal subunit (grey) with the ribosomal proteins surrounding the tunnel exit. Conservation of tunnel exit proteins are outlined in the table below and are shown in color code (green conserved in all kingdoms of life; blue exclusively found in eukaryotes). (C) Crystal structure of $E$. coli $\mathrm{TF}$ based on the data from [81] together with the outline of the linear domain arrangement. TF domains are shown in red ( $\mathrm{N}$-terminal tail domain with the conserved signature motif mediating ribosome binding), green (PPIase head

domain) and in blue (C-terminal domain forming the body of the molecule). The tail together with the body forms a large cradle-like structure indicated by the dotted lines. (D) Model of the TF mechanism. Ribosome-associated TF (red) interacts with an unfolded nascent polypeptide by transient hydrophobic contacts within the TF cradle (1). As soon as a complete domain is synthesized, folding can occur underneath TF (2) causing the detachment of TF from its substrate and perhaps the dissociation of TF from the ribosome (3). TF rebinds to the ribosome when a new stretch of the newly synthesized polypeptide becomes exposed at the exit tunnel to promote the folding of the next domain. The newly synthesized protein is released in an intermediate or almost completely folded state and can finish its folding program either spontaneously or by employing downstream acting chaperones (4). Ribosome-bound TF awaits the next translation cycle (5).

central anchor point for the bacterial chaperone TF [15] and for the SRP particle [16-18]. Interestingly, quite a number of additional factors, unrelated to chaperones or targeting factors, can associate with ribosomes and the growing polypeptide during the early stages of protein biosynthesis. These additional factors mostly represent modifying enzymes such as the essential methionine aminopeptidase (MetAP) in pro- and eukaryotes, which removes the $\mathrm{N}$-terminal methionine from nascent chains, and the N-terminal acetylases of yeast and higher organisms. These enzymes modify the nascent polypeptides co-translationally as soon as they expose their $\mathrm{N}$-termini on the ribosomal surface. Thus, it is tempting to speculate that these enzymes could also occupy docking sites close to the tunnel exit. Indeed, it was suggested recently that the aminopeptidase from Mycobacterium tuberculosis might interact via a PXXP motif with the SH3-domain of the conserved L24 protein [19], the protein located in the vicinity of to the chaperone docking site L23. It will be exciting to unravel how these different enzymes, chaperones and targeting factors, present at the interface between the ribosome and the crowded cellular environment, are coordinated in their order and mode of action to ensure the correct fate of a newly synthesized protein.

\section{When does a protein start folding?}

This question cannot yet be answered in a general way since thus far only a few model proteins have been inves- 
tigated and the folding program may differ for each species of newly synthesized protein. Clearly, a polypeptide chain can finish its folding program only after synthesis and release from the ribosome. Proteins are synthesized by the ribosome in a vectorial manner from the $\mathrm{N}$-terminus towards the $\mathrm{C}$-terminus. Thus the $\mathrm{N}$-terminal part of the polypeptide chain already exits the ribosomal tunnel while the C-terminal portion is still being synthesized. Little is known about the conformation of the nascent polypeptide in the tunnel. The diameter of the tunnel could allow for a peptide to acquire a helical structure, but it seems unlikely that significant protein folding can occur beyond such a conformation [5]. Using a different experimental approach, a recent Cryo-EM study suggests that small segments of a stalled nascent polypeptide could potentially be located within an expanded tunnel, perhaps in a globular conformation [20]. Yonath and colleagues have also recently described a crevice emanating from an early position along the ribosomal exit tunnel of the eubacterial ribosome, which they claim could be large enough to accommodate some secondary structural folds of a nascent chain and contribute to the co-translational folding of the protein or regulation of the associated ribosome [9]. However, the biological significance and universal validity of these findings for protein folding is unclear and must be investigated further.

Several studies provided evidence that protein folding can start co-translationally during biosynthesis as soon as a polypeptide leaves the ribosomal exit tunnel [21-24]. This concept was exemplified in vivo by experiments demonstrating the co-translational protein folding in the cytosol of pro- and eukaryotic cells using the Semliki Forest Virus C-protein as a model system [24]. The Cprotein is the $\mathrm{N}$-terminal portion of a multi-polypeptide precursor that auto-catalytically cleaves itself of its residual polypeptide as soon as it is folded co-translationally during ongoing synthesis.

The overall proportion of all newly synthesized proteins that start their folding program co-translationally remains unclear. In fact, two studies discuss fundamental differences that might exist between pro- and eukaryotic organisms in their capacities to fold proteins co-translationally. This assumption was based on the finding that in bacteria, but not in reticulocyte lysate, the folding of newly synthesized large model proteins occurs mainly post-translationally, as shown for the artificial Ras-DHFR fusion protein [25] and firefly luciferase [26]. Therefore, it was suggested that rapid and efficient folding of multi-domain proteins occurs co-translationally in the eukaryotic system, while the bacterial system lacks the capacity for folding these model proteins cotranslationally. However, it is not known whether this assumption holds true for the bulk of bacterial multidomain proteins. A protein's preferred mode of folding may depend on the particular folding kinetics, domain sizes and environmental conditions in which the protein is being synthesized. The action of chaperones might not only be required to promote the folding of a newly synthesized protein by minimizing aggregation or premature degradation of folding intermediates, but may also be important to prevent premature folding of nascent polypeptide chains until sufficient sequence information is provided such that the newly made protein can form its correct domain structures. It is generally assumed that the sequential co-translational folding of domains during biosynthesis could be of particular advantage for the folding of large multi-domain proteins because it limits possible unproductive inter- and intramolecular interactions during the early folding steps $[1,21]$. This is particularly important since massive macromolecular crowding in the cell $(200-300 \mathrm{mg} / \mathrm{ml}$ cytosolic protein concentration) and high local concentrations of nascent polypeptides increase aggregation and interfere with productive folding in vivo [27].

\section{Chaperones guide the folding of newly synthesized proteins}

A common feature of all chaperones is the stoichiometric and transient binding to folding intermediates. Chaperones prevent misfolding and aggregation by binding to hydrophobic patches of non-native proteins, thereby influencing the partitioning between productive and unproductive folding steps. Importantly, chaperones are not part of the final structures of the folded proteins. Instead substrates are released from the chaperones, thereby providing non-native proteins with a new opportunity for productive folding [28-30]. Most chaperones are conserved members of the heat shock protein (Hsp) families Hsp100, Hsp90, Hsp70, Hsp60, Hsp40 and the small Hsps (the numbers indicate the typical molecular mass of the family members). In contrast, some other chaperones are not maintained throughout evolution such as the TF, which is present only in bacteria and chloroplasts, or the Hsp70/40 pair Ssz/Zuotin (Zuo) which is found exclusively in eukaryotes and does not exist in the prokaryotic world.

Chaperones that assist the folding of cytosolic proteins can be classified into two subgroups according to their localization [1, 31, 32]. The first class is composed of soluble cytosolic chaperones that associate co- and posttranslationally with newly synthesized proteins. They predominantly include members of the Hsp60 and Hsp70 families. The second class of chaperones assemble directly at the ribosomal exit tunnel, serving as the molecular threshold which must be traversed by newly synthesized proteins as they enter into their cellular home. The key features of the second category of chaperones are their attachment to the translation machinery and their interac- 
tion with the nascent polypeptide during protein biosynthesis. The coupling of protein biosynthesis with protein folding via ribosome-associated chaperones seems to be an evolutionarily ubiquitous principle, although the specific types of chaperones utilized by different organisms in this process are seemingly unrelated.

\section{Ribosome-associated chaperones: key players in the folding pathways of newly synthesized proteins}

Ribosome-tethered chaperones have primary access to newly synthesized proteins during biosynthesis. Thus it is believed that these chaperones are of particular importance for a polypeptide to enter into its correct folding pathway. Remarkably, neither sequence nor structural homology exists between the different ribosome-associated chaperones found in prokaryotes and eukaryotes. Nevertheless, they do show intriguing functional similarities in the way that they interact with ribosomes and nascent polypeptides. They all are abundant proteins that associate with the large ribosomal subunit in a 1: 1 stoichiometry, and they can be crosslinked to a large variety of nascent polypeptides [33-36]. The presence and absence of nascent chains alters the interaction of these chaperones with the ribosome, as indicated by an increased salt resistance of the chaperone-ribosome interaction in the presence of a nascent chain.

\section{Eukaryotic ribosome-associated chaperones and their role in protein folding}

To date, the yeast Saccharomyces cerevisiae is the best-studied eukaryotic system. In yeast, two different ribosome-associated systems are found which could act co-translationally in the protein folding program: the $\mathrm{Ssb} / \mathrm{Ssz} / Z u o-t r i a d$ and NAC (fig. 2B).

The first system is an Hsp70/Hsp40-based chaperone system containing two Hsp70 members, namely Ssb and Ssz, and Ssz's Hsp40 co-chaperone Zuo. Ssz and Zuo form the ribosome-associated complex termed RAC (fig. 2B) [37]. RAC together with Ssb forms the so-called ribosomal chaperone triad [38]. Inactivation of the yeast chaperone triad through deletion of either one or all of its members leads to the appearance of three phenotypes: salt sensitivity, cold sensitivity, and hypersensitivity to aminoglycosides such as paromomycin that block ribosomal protein synthesis and impair translational fidelity $[37,39,40]$. The specific function of the ribosome-associated triad and the molecular basis of the observed phenotypes of the yeast triad mutants are still unknown, but some clues are beginning to emerge. It was previously observed that cells lacking at least Ssb have an increased uptake of components from the surrounding medium, leading to various pleiotropic effects [41]. A recent, more detailed study finds that cells lacking either Ssb or Zuo have an increased uptake of cations from their surrounding environment, correlating with decreased activity of plasma membrane ion transporters [42]. Such pleiotropic defects could arise as a result of either the increased level of translational misreading occurring in these strains in the presence of aminoglycosides or the absence of a required chaperone activity provided by the triad, leading to the production of defective gene products that could either directly or indirectly disrupt cellular processes such as membrane transport.

Although the exact role of the triad in co-translational protein folding remains elusive, it is likely that as a unit the triad functions as a bona fide chaperone system since all of its members belong to classical chaperone families, notwithstanding some very interesting features which make such a chaperone system quite unique. For example, it has now been reported that Ssz does indeed regulate the activity of Zuo, and it does so independent of Ssz's ATPase activity [43]. With this assistance from Ssz, Zuo can then specifically and efficiently stimulate the ATPase activity of Ssb. It is also noteworthy that unlike many chaperones that are induced in response to cellular stress, the core Hsp70-Ssb protein of the triad is not upregulated in response to stress, but rather, in accordance with a role for Ssb in protein folding at the ribosome, it is co-regulated with the ribosomal machinery [44]. The likely role of the triad as a chaperone is further underscored by recent findings showing that the prokaryotic ribosome-bound chaperone TF, when expressed in S. cerevisiae, can substitute in vivo for the functions of the chaperone triad $[45,46]$. TF restores the ability of yeast cells lacking the $\mathrm{Ssb} / \mathrm{Ssz} /$ Zuo triad to grow in the presence of high concentrations of salts or aminoglycosides. TF rescue of these cells is dependent upon its ability to bind to ribosomes, where TF apparently binds to yeast ribosomes at $\mathrm{L} 25$, the yeast homolog of the E. coli $\mathrm{L} 23$ docking site. Furthermore, like Ssb, TF can crosslink to nascent chains generated in a yeast translation extract in vitro. Taken together, these data highlight redundant functions for otherwise non-homologous players in the protein folding program at the ribosome.

Until recently, it was thought that the $\mathrm{Ssb} / \mathrm{Ssz} /$ Zuo system is a specific adaptation of fungi since no orthologs were known in higher eukaryotic cells. However, very recently two mammalian homologs of the triad members were discovered showing that these ribosome-associated chaperones were maintained throughout eukaryotic evolution $[47,48]$. The mammalian Zuo homolog MPP11 associates with ribosomes and complements the phenotype of a Zuo deletion in yeast. Moreover, Rospert and co-workers found that MPP1 1 forms a stable complex with Hsp70L1, a mammalian Ssz homolog, indicating the conservation of the entire RAC complex. Interestingly, mammalian 
A
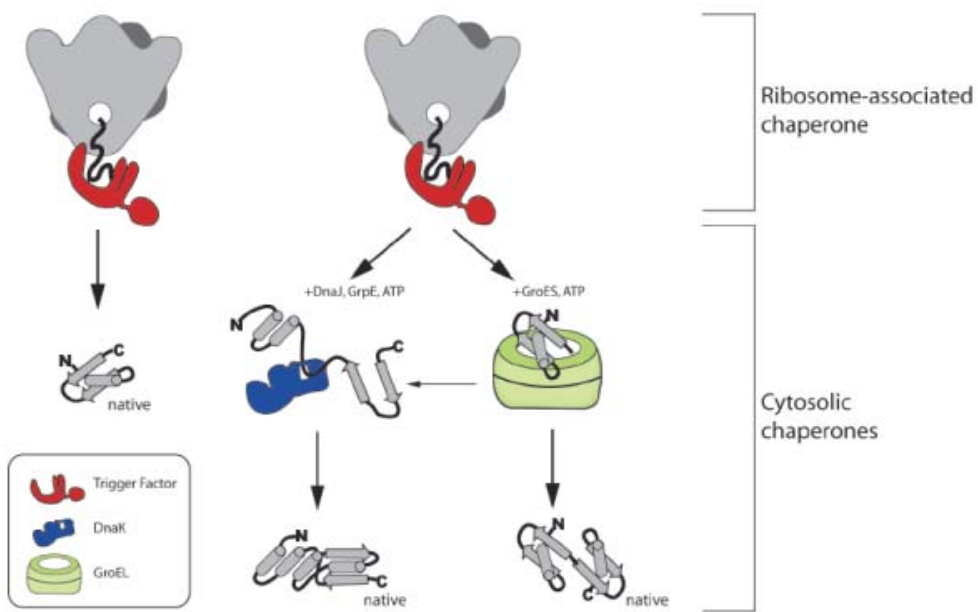

B

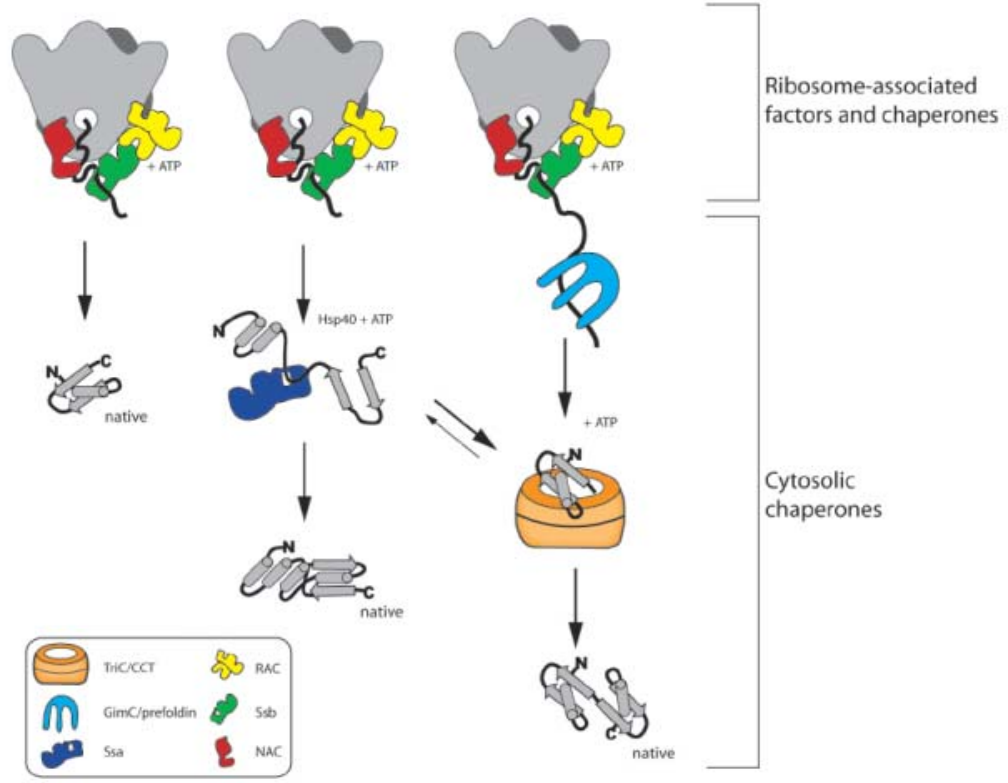

Figure 2. Models of chaperone-assisted folding pathways in the cytosol. (A) In prokaryotes, TF interacts with nascent polypeptides during biosynthesis to promote cotranslational folding processes. Beyond the interaction of newly made proteins with $\mathrm{TF}$, some proteins may fold autonomously to their native state, whereas many others require the additional downstream action of the Hsp70 DnaKsystem (acting in concert with its co-chaperones DnaJ and GrpE under ATP consumption) and/or the Hsp60 GroEL system (acting in concert with its co-chaperone GroES under ATP consumption). (B) In eukaryotes the situation is more complex and exemplified by showing the yeast scenario. Two systems act on nascent polypetide chains on the ribosome: the nascent chain-associated complex NAC and the Hsp70/40-based system Ssb/Ssz/Zuo. Similar to the prokaryotic situation many proteins need further support by cytosolic chaperones of the Hsp70 (Ssa) or Hsp60 (TriC/CCT) category to allow for efficient folding. GimC/prefoldin is a chaperone required to fold actin and tubulin.
MPP11 expressed in yeast constitutes a functional Hsp40 partner for the cytosolic Hsp70-Ssa (fig. 2B) but does not cooperate with the ribosome-bound Ssb. Obviously MPP11 does not require a ribosome-bound Hsp70 partner and may rather target cytosolic Hsp70 to the nascent chain. This assumption is supported by earlier findings of Welch and co-workers showing that cytosolic Hsp70 associates with ribosome-nascent chain complexes and crosslinks to nascent polypeptides [49]. Taken together, if the RAC of higher eukaryotes can work in concert with a cytosolic Hsp70 partner, these observations are consistent with the fact that to date no ribosome-tethered Hsp70Ssb ortholog has been detected in mammals. Although more experimental work is required to clarify this, it is tempting to speculate that in metazoans the mechanistic scenario of this ribosome-based Hsp40/70 based system is modified, albeit the general principle is maintained: the system works on nascent chains during biosynthesis.

In addition to the triad, the protein complex NAC associates with both ribosomes and nascent chains (fig. 2B). NAC is conserved throughout the eukaryotic world from yeast to humans [50-52], where it is present as a heterodimer composed of an $\alpha$ - and $\beta$-subunit. NAC is also found in archaea; however, here the $\beta$-subunit is absent, and a homodimer is formed by two $\alpha$ components [53, 54]. Although a clear chaperone role for NAC in cells is yet to be demonstrated, it is considered here since NAC has very early access to nascent polypeptides, suggesting that at some level NAC could contribute either directly or indirectly to the folding program of these chains. In 
yeast, the deletion of the genes encoding NAC results in no obvious phenotype; however, when the $\alpha$-subunit alone is present a growth defect could be observed [55]. The reason for this effect is unclear. NAC has been described to be involved in mitochondrial import of precursor proteins by having a stimulatory effect on protein targeting in vitro [51]. Moreover, it was proposed that NAC has a role in controlling the correct translocation to the ER. It has been shown that in the absence of NAC, ribosomes translating non-secretory polypeptides inappropriately interacted with translocation sites on ER membranes [56, 57]. However, owing to the lack of in vivo data to support this finding, this function of NAC is not generally accepted. Although the in vivo function of NAC remains obscure, NAC's general significance is emphasized by the embryonic lethality of NAC mutants in mice, nematodes and fruit flies [58-60].

\section{Interaction of eukaryotic factors with ribosomes and nascent polypeptides}

Among the members of the chaperone triad, strikingly only Ssb crosslinks to nascent polypeptide chains, and this critically depends on the presence of the Zuo/Ssz (RAC) complex, which is believed to serve as the cochaperone of Ssb [34, 38, 40]. Zuo tethers Ssz to the ribosome, whereas Ssb contacts the ribosome autonomously [34, 37, 39]. NAC's association with the ribosome in eukaryotes is exclusively mediated by its $\beta$-subunit. Deletion of the N-terminal 11 amino acid residues of $\beta N A C$ in yeast results in the loss of ribosome association and cellular mislocalization [61]. Whereas the $\beta$-subunit is responsible for the association of NAC with the ribosome, both subunits were shown to crosslink to very short nascent polypeptide chains [36].

Structural data about the triad and the eukaryotic NAC complex is still lacking, but very recently the crystal structure of the archaea $\alpha$ NAC homolog, aeNAC, was published [53]. Consistent with protein domain predictions, the aeNAC structure reveals two major structural features: the NAC domain and a UBA (ubiquitin-associated) domain. The NAC domain shapes a novel protein fold responsible for homodimerization of aeNAC and is therefore also likely to be responsible for heterodimerization of the complex in eukaryotes. UBA domains, forming a compact three-helix bundle with a conserved hydrophobic patch on their surface, were initially identified in proteins such as ubiquitin ligases and other proteins linked to the ubiquitin/proteasome pathway [62]. The eukaryotic $\alpha \mathrm{NAC}$, but not $\beta \mathrm{NAC}$, also contains such a UBA domain. The presence of a UBA domain in NAC is intriguing since the published functions of this domain are multifaceted. UBA domains can clearly interact with various ubiquitin moieties (for review, see [63]). In this way, proteins containing UBA domains can contribute to the folding program of a nascent polypeptide by providing a direct link to the ubiquitin/proteasome pathway that can clear cells of misfolded proteins. Indeed, co-translational ubiquitination of nascent chains was previously described [64, 65]. Alternatively, UBA domains have also been described to contribute to the dimerization of complex molecules and to protein-protein interactions in general (see [63] and references therein). The functional relevance of the UBA domain present in the NAC complex is therefore a topic of great interest.

\section{Prokaryotic ribosome-associated chaperone TF}

Although nature has clearly employed the strategy of associating chaperones with ribosomes across all kingdoms (fig. 2A, B), the structural and mechanistic principles of these proteins appear to differ. The ribosome-associated chaperone TF is found in almost all bacteria and in chloroplasts but is absent from the cytosol of archaea and eukaryotes. As the best-studied ribosome-associated chaperone, TF is discussed in detail below as an example of how protein synthesis and folding can proceed efficiently as a coupled process.

\section{In vivo role of $\mathrm{TF}$ in protein folding}

The role of TF in protein folding was elucidated a few years ago by investigating an $E$. coli strain that lacks the TF encoding gene tig $[66,67]$. Surprisingly, these cells exhibited neither any obvious growth phenotype nor any misfolding of newly synthesized proteins. Most likely this observation can be accounted for by the subsequent compensatory induction of the heat shock response leading to increased steady state levels of other chaperones, including DnaK, and proteases in these $\Delta$ tig cells [68]. The simultaneous deletion of the tig and dnaK genes leads to synthetic lethality at $30^{\circ} \mathrm{C}$ and above $[66,67]$. Thus, either TF or DnaK is sufficient for cell viability at permissive temperatures, but the loss of both leads to cell death, indicating that these two chaperones cooperate in the folding of newly synthesized polypeptides. In wildtype $E$. coli cells about $5-18 \%$ of newly synthesized proteins interact transiently with DnaK (fig. 2A). This level increased two- to three-fold (26-36\%) in the absence of $\mathrm{TF}$, indicating that upon the loss of TF the cells make more intensive use of the DnaK chaperone [66, 67]. Thus DnaK may serve as a potent supporting system in the absence of TF. Moreover, when both TF and DnaK are absent, recent studies have demonstrated that some cells can cope with this loss by utilizing SecB and GroEL/ES chaperones as further backup systems [69-71].

The depletion of DnaK in the absence of TF leads to the massive aggregation of more than 300 cytosolic proteins in E. coli: approximately $10 \%$ of total cellular cytosolic 
proteins are aggregation-prone at $37^{\circ} \mathrm{C}$, whereas only about $1 \%$ of the cytosolic proteins aggregated in cells depleted of only DnaK [68]. While the total amount of aggregated proteins differed significantly in DnaK-depleted $\mathrm{tig}^{+}$and $t i g^{-}$cells, the aggregation-prone protein species in these cells were similar, suggesting that TF and DnaK share overlapping substrate pools. The aggregated protein species found in cells lacking TF and DnaK range in their molecular weights from 16 to $167 \mathrm{kDa}$ and include many essential proteins such as the elongation factor EF-Tu and the RNA-polymerase subunit RpoB, which could explain the synthetic lethality of $\Delta t i g \Delta d n a K$ mutant cells. These aggregation-prone proteins do not show any common features regarding $\mathrm{pI}$ or their specific secondary structural elements, with the exception of a number of large multi-domain proteins. The enrichment of large-sized multi-domain proteins $(>60 \mathrm{kDa})$ among those folding-incompetent protein species indicate that the size or domain composition of a protein is one crucial factor that determines a protein's chaperone requirement during de novo folding.

How could TF and DnaK, which work by completely different mechanisms, cooperate in the chaperoning of a newly made protein? A possible explanation relates to the finding that TF and DnaK reveal overlapping features of their substrate binding motifs. The TF binding motif consists of eight amino acids enriched in basic and aromatic residues. The placement of such residues within this motif seems to be arbitrary [72]. DnaK recognizes a related, but not identical, binding motif. This chaperone recognizes a short stretch of five amino acid residues with hydrophobic character, among which leucine is especially favored. This hydrophobic core is flanked by positively charged amino acids, whereas negative charges are excluded [73, 74]. TF and DnaK thus have an affinity for hydrophobic and positively charged stretches within proteins [72, 75]. The redundancy in binding specificity of these two chaperones, despite their different mechanisms of action, allows for the protection of similar hydrophobic stretches in unfolded protein species and therefore promotes the folding of the same substrate pool.

\section{TF structure and ribosomal localization}

E. coli $\mathrm{TF}$ consists of 432 amino acids with a molecular weight of $48 \mathrm{kDa}$ and is composed of three domains (fig 1C). The N-terminal domain of TF (aa 1-148) is necessary and sufficient for the specific binding of TF to ribosomes [76]. Within this N-domain, a highly conserved GFRXGXXP motif termed the TF signature is essential for its ribosome interaction [15]. Mutation in this TF signature motif results in a strong ribosome-binding deficiency. Importantly, the docking of TF to the ribosome via this signature motif is essential for its interaction with nascent chains. L23, one of the universally con- served proteins surrounding the exit site of the ribosome, serves a docking site for TF. Point mutations of residues on the cytoplasmically exposed surface of L23 decrease TF's ribosome binding and lead to synthetic lethality at $37^{\circ} \mathrm{C}$ in cells lacking the DnaK backup system. The second TF domain (aa 149-245) carries a catalytic activity as a peptidyl-prolyl cis/trans isomerase (PPIase) and has homology to the FKBP (FK506 binding protein) type of PPIases. The contributions of this domain to TF's function in de novo folding of proteins is puzzling since mutations in this domain, either point mutations diminishing the catalytic PPIase activity or deletion of the entire domain, still display chaperone activity in vivo comparable to wild type TF [71, 77-79]. The C-terminal domain (aa 246-432), which constitutes nearly half of the TF protein, reveals no homology on the amino acid level to any other protein [80]. Deletion of this domain severely decreases TF chaperone activity in vitro and in vivo [71, $77,78]$, indicating that this domain itself participates in substrate binding.

Very recently, the crystal structure of E. coli TF [81-84], as well as that of an $\mathrm{N}$-terminal TF fragment bound to Haloarcula marismortui 50S, were solved [81]. This structural study revealed the unusual extended shape of the TF molecule (fig. 1C). The N-terminal ribosomebinding domain builds the tail of the molecule, whereas the second domain in the linear sequence of TF harboring the PPIase activity is located at the head of the molecule, and is connected to the tail with an 80 - $\AA$-long linker that extends along the back of the TF. The $\alpha$-helical C-terminal domain inserts between the first and the second domain in the three-dimensional structure to build the back and the arms of TF. The remarkable result is a large cavity formed between the N-terminal ribosome binding tail and the $\mathrm{C}$-terminal arms. The interior of this cradle is characterized by the exposure of numerous hydrophobic side chains offering multiple contact sites for an unfolded polypeptide chain.

\section{Model for the TF mechanism}

The accurate placement of full-length TF on the ribosome could be deduced based on the crystal structure of a complex between the $E$. coli $\mathrm{N}$-domain of TF and the $H$. marismortui $50 \mathrm{~S}$ subunit, thus providing new insights into how TF may promote the folding of newly made proteins during biosynthesis [81]. By binding to the ribosome via L23, TF hunches over the polypeptide exit of the ribosome and exposes the sticky, hydrophobic inner face of its cradle towards the area where the nascent polypeptides emerges from the ribosome. These structural insights, in agreement with existing evidence demonstrating the role of the cradle as the major substrate binding site of TF [78], suggest that TF provides a shielded folding environment for nascent polypeptides 
with its hydrophobic cradle, ideally positioned on top of the ribosomal exit site for the capture of emerging chains. Newly made proteins are synthesized into the TF cradle, protected from unproductive interactions such as aggregation or premature degradation (fig. 1D).

What is the fate of a nascent chain that is captured underneath TF? Two alternative models are plausible which are not mutually exclusive and may depend on the particular substrate synthesized at the ribosome. The first model suggests that the nascent chain stays inside the cradle, and this allows for the formation of a folded core or domain as soon as sufficient folding information is synthesized (fig. 1D). In the second model, the nascent chain does not fold underneath TF, but rather TF serves as a protective shield preventing unproductive interactions or untimely degradation of the nascent chain.

In support of the first model, the crystal structure of TF indicates that the cradle can accommodate protein domains of at least $15 \mathrm{kDa}$. The average size of a domain is between 100 and 130 aa, corresponding to 11 and 14 $\mathrm{kDa}$, respectively. It is thus tempting to speculate that the TF cradle would be ideally sized to support the cotranslational domain-wise folding of many large multidomain proteins (fig. 1D). This assumption is in agreement with the finding that the isolated cradle domain, corresponding to a TF molecule that lacks the PPIase domain, constitutes the major substrate binding site of TF and exhibits almost wild-type-like chaperone activity in vivo and in vitro $[71,78]$. A recent study showed that when TF was added to an in vitro $E$. coli-based translation system where the model substrate luciferase was synthesized, folding was delayed, yet the total yield of active protein was greater than in the absence of TF [26]. However, it should be noted that firefly luciferase has two domains of approximately 22 and $40 \mathrm{kDa}$ and could perhaps belong to a class of substrates that, rather than being folded underneath $\mathrm{TF}$, are protected from premature unproductive folding.

It is unclear whether, in either scenario, the nascent chain traverses the arms towards the PPIase domain by multiple rounds of binding and release within TF. In both models TF would interact with its substrate via multiple transient hydrophobic contacts of low affinity, and such interactions may initially prevent the immediate folding of the nascent chain. This is in agreement with a recent finding that TF retards the folding of nascent polypeptides both in vivo and in vitro [26]. Furthermore, the question remains, how is the interaction between TF and the nascent polypeptide is disrupted? Perhaps, as soon as a nascent domain folds, hydrophobic interactions between the substrate and TF are lost and the folded domain is then released from the chaperone. It is also feasible that the folding process may generate a mechanical force that strips TF from the emerging polypeptide chain. Intensive investigation is currently underway to reach the ambitious goal of dissecting the mechanistic pathway and kinetics of TF action on nascent substrates.

\section{Integration of ribosome-associated chaperones into a robust cytosolic chaperone network}

Although the mechanistic principles of protein folding at the ribosome have not been clarified in detail, it is known that once different nascent polypeptides leave the ribosome, they may utilize different paths along an integrated chaperone network in order to achieve their mature folded status. In bacteria, TF potentially interacts with all nascent chains emerging from the ribosomal exit tunnel (fig. 2A) [35, 85, 86]. After release from TF, the nascent polypeptide can start or continue its folding to the native state $[26,87]$. Small proteins that show fast folding kinetics in vitro may not require further guidance from additional chaperones to fold into their native structures. In contrast, multi-domain proteins have been shown, at least in vitro, to fold more slowly and to become trapped in non-native aggregation-prone conformations. Such proteins may need further assistance by ATP-consuming chaperones at downstream steps along the folding pathway and would constitute typical substrates for the DnaK (Hsp70) and GroEL (Hsp60) chaperone systems $[1,31]$. In the bacterial cell, the majority of the GroEL chaperone machinery associates with newly synthesized proteins almost exclusively after their release from the ribosome, functionally distinguishing GroEL from the DnaK chaperone, which associates with both nascent and released polypeptides [66-68, 88, 89]. Based on the observation that GroEL/ES can rescue cells from depletion of both TF and DnaK [70, 71], it was suggested that in the absence of these factors GroEL/ES might be able to interact in a direct way with nascent polypeptide chains. New experiments now reveal that GroEL can indeed stably and productively interact co-translationally with nascent model substrates that are bound to the ribosome in vitro [90], adding to the general complexity of chaperone factors associated with the ribosome. Under most cellular conditions, it is agreed that the location of TF immediately adjacent to the ribosome exit tunnel places it upstream of DnaK and GroEL with regard to nascent chain interaction [15, 81]. Downstream of TF, DnaK and GroEL form a functional network in which substrates may be transferred bidirectionally from one chaperone to the other, through a soluble intermediate [91].

The situation in the eukaryotic cell is similar (fig. 2B) in that chaperones and factors which are directly bound to the ribosome, namely NAC, RAC and Ssb in yeast, would be the first to encounter emerging nascent polypeptides. In contrast to the bacterial scenario where only the TF chaperone interacts with nascent polypeptides, a multitude of ribosome-associated factors discussed herein 
are likely to interact with growing nascent chains in the eukaryotic cell. However, it is unclear whether these factors interact in hierarchical manner with nascent chains and whether these factors display specific functions for a particular subset of nascent polypeptides. One pressing current goal of the field is to investigate the hierarchical activities of these molecules by trying to determine where exactly NAC, RAC and Ssb bind near the ribosomal exit tunnel.

Downstream of the ribosome-associated factors, cytosolic chaperone players such as the eukaryotic GroEL counterpart TriC/CCT [92-94], the Hsp70-system [95] and GimC/prefoldin, a chaperone specific for the folding of actin and tubulin [96, 97], are further required for de novo protein folding. Like their prokaryotic counterparts, the bidirectional shuttling of substrates between various different cytosolic chaperones also occurs in eukaryotes. As not every chaperone can appropriately handle every substrate, and indeed the proper folding program of a protein is likely to require a different sequence of productive interactions, the different chaperones sample the various substrates to some degree so that the appropriate clients are selected. Using an in vitro coupled transcription-translation and photo-crosslinking system, it has now been demonstrated that emerging polypeptides interact with specific subunits of the oligomeric TriC/CCT chaperonin co-translationally, and the nature of these interactions changed in a nascent chain length-dependent manner [92]. This suggests that differential substrate specificity exists even among the different subunits of such a chaperone machine, and when the local concentration of a potential substrate is high, in this case with cytosolic TriC/CCT positioned near the nascent chain which exits the ribosomal tunnel, TriC/CCT can sample a multitude of emerging potential substrates in search of its proper client proteins. In this way the eukaryotic system, employing hetero-oligomeric TriC/CCT, has obtained an additional level of complexity over the homo-oligomeric GroEL chaperonin of prokaryotes.

\section{Perspectives}

As summarized here, recent advances regarding the role of ribosome-associated factors in protein folding bring us closer to understanding how cells utilize an ensemble of different proteins to coordinate the processes of protein synthesis and folding. It is becoming clear that the concerted effort of ribosome-associated and downstream cytoplasmic factors is in many cases required to achieve properly folded and functional molecules. The eukaryotic and prokaryotic worlds have used both similar (chaperonins and DnaK homologs) and unrelated (bacterial TF and the yeast $\mathrm{Ssb} / \mathrm{Ssz} / \mathrm{Zuo}$ triad) strategies to achieve the same functional goal. It is with great excitement that we look forward to the next challenges in this field, which include the detailed mapping of the docking sites of ribosomeassociated factors on the ribosome, clarification of the mechanistic principles of ribosome-bound chaperones, insight into the hierarchical processes that govern protein folding in the cell, and genetic or environmental factors which allow the re-programming of these processes.

Acknowledgements. We thank the members of the Deuerling and Bukau labs for helpful discussions, in particular we thank Frieder Merz for his help in the preparation of figure 1C. This work was supported by grants from the Human Frontier in Science Program (HSFP) and the DFG to E.D., a Heisenberg fellowship to E.D. and an Alexander von Humboldt fellowship to R.D.W.

1 Bukau B., Deuerling E., Pfund C. and Craig, E. A. (2000) Getting newly synthesized proteins into shape. Cell 101: 119-122

2 Ban N., Nissen P., Hansen J., Moore, P. B. and Steitz, T. A. (2000) The complete atomic structure of the large ribosomal subunit at 2.4 Å resolution. Science 289: 905-920

3 Schluenzen F., Tocilj A., Zarivach R., Harms, J. Gluehmann M., Janell D. et al. (2000) Structure of functionally activated small ribosomal subunit at 3.3 angstroms resolution. Cell 102: 615-623

4 Wimberly B. T., Brodersen D. E., Clemons W. M. Jr, MorganWarren R. J., Carter A. P., Vonrhein C. et al. (2000) Structure of the 30 S ribosomal subunit. Nature 407, 327-339

5 Nissen P., Hansen J., Ban N., Moore P. B. and Steitz T. A. (2000) The structural basis of ribosome activity in peptide bond synthesis. Science 289: 920-930

6 Ban N., Nissen P., Hansen J., Capel M., Moore P. B. and Steitz T. A. (1999) Placement of protein and RNA structures into a 5 A-resolution map of the 50S ribosomal subunit. Nature 400: 841-847

7 Hardesty B. and Kramer G. (2001) Folding of a nascent peptide on the ribosome. Prog. Nucleic Acid Res. Mol. Biol. 66: 41-66

8 Spahn C. M., Beckmann R., Eswar N., Penczek P. A., Sali A., Blobel G. et al. (2001) Structure of the 80S ribosome from Saccharomyces cerevisiae-tRNA-ribosome and subunit-subunit interactions. Cell 107: 373-386

9 Amit M., Berisio R., Baram D., Harms J., Bashan A. and Yonath A. (2005) A crevice adjoining the ribosome tunnel: hints for cotranslational folding. FEBS Lett. 579: 3207-3213

10 Baram D. and Yonath A. (2005) From peptide-bond formation to cotranslational folding: dynamic, regulatory and evolutionary aspects. FEBS Lett. 579: 948-954

11 Nakatogawa H. and Ito K. (2002) The ribosomal exit tunnel functions as a discriminating gate. Cell 108: 629-636

12 Gong F. and Yanofsky C. (2002) Instruction of translating ribosome by nascent peptide. Science 297: 1864-1867

13 Tenson T. and Ehrenberg M. (2002) Regulatory nascent peptides in the ribosomal tunnel. Cell 108: 591-594

14 Fang P., Spevak C. C., Wu C. and Sachs M. S. (2004) A nascent polypeptide domain that can regulate translation elongation. Proc. Natl. Acad. Sci. USA 101: 4059-4064

15 Kramer G., Rauch T., Rist W., Vorderwülbecke S., Patzelt H., Schulze-Specking A. et al. (2002) L23 protein functions as a chaperone docking site on the ribosome. Nature 419: 171-174

16 Gu S. Q., Peske F., Wieden H. J., Rodnina M. V. and Wintermeyer W. (2003) The signal recognition particle binds to protein L23 at the peptide exit of the Escherichia coli ribosome. RNA 9: $566-573$

17 Pool M. R., Stumm J., Fulga T. A., Sinning I. and Dobberstein B. (2002) Distinct modes of signal recognition particle interaction with the ribosome. Science 297: 1345-1348

18 Ullers R. S., Houben E. N., Raine A., ten Hagen-Jongman C. M., Ehrenberg M., Brunner J. et al. (2003) Interplay of signal recognition particle and trigger factor at L23 near the nascent 
chain exit site on the Escherichia coli ribosome. J. Cell Biol. 161: 679-684

19 Addlagatta A., Quillin M. L., Omotoso O., Liu J. O. and Matthews B. W. (2005) Identification of an SH3-binding motif in a new class of methionine aminopeptidases from Mycobacterium tuberculosis suggests a mode of interaction with the ribosome. Biochemistry 44: 7166-7174

20 Gilbert R. J., Fucini P., Connell S., Fuller S. D., Nierhaus K. H., Robinson C. V. et al. (2004) Three-dimensional structures of translating ribosomes by Cryo-EM. Mol. Cell 14: 57-66

21 Netzer W. J. and Hartl F. U. (1997) Recombination of protein domains facilitated by co-translational folding in eukaryotes. Nature 388: 343-349

22 Frydman J., Erdjument-Bromage H., Tempst P. and Hartl F. U. (1999) Co-translational domain folding as the structural basis for the rapid de novo folding of firefly luciferase. Nat. Struct. Biol. 6: 697-705

23 Kolb V. A., Makeyev E. V. and Spirin A. S. (2000) Co-translational folding of an eukaryotic multidomain protein in a prokaryotic translation system. J. Biol. Chem. 275: 16597-16601

24 Nicola A. V., Chen W. and Helenius A. (1999) Co-translational folding of an alphavirus capsid protein in the cytosol of living cells. Nat. Cell Biol. 1: 341-345

25 Netzer W. J. and Hartl F. U. (1998) Protein folding in the cytosol: chaperonin-dependent and -independent mechanisms. Trends Buiochem. Sci. 23: 68-73

26 Agashe V. R., Guha S., Chang H. C., Genevaux P., Hayer-Hartl M., Stemp, M. et al. (2004) Function of trigger factor and DnaK in multidomain protein folding: increase in yield at the expense of folding speed. Cell 117: 199-209

27 Zimmerman S. B. and Trach S. O. (1991) Estimation of macromolecule concentrations and excluded volume effects for the cytoplasm of Escherichia coli. J. Mol. Biol. 222: 599-620

28 Buchner J. (1996) Supervising the fold: functional principles of molecular chaperones. FASEB J. 10: 10-19

29 Bukau B. and Horwich A. L. (1998) The Hsp70 and Hsp60 chaperone machines. Cell 92: 351-366

30 Ellis R. J. and Hartl F. U. (1999) Principles of protein folding in the cellular environment. Curr. Opin. Struct. Biol. 9: 102-110

31 Hartl F. U. and Hayer-Hartl M. (2002) Molecular chaperones in the cytosol: from nascent chain to folded protein. Science 295: $1852-1858$

32 Frydman J. (2001) Folding of newly translated proteins in vivo: the role of molecular chaperones. Annu. Rev. Biochem. 70: 603-647

33 Hesterkamp T., Hauser S., Lutcke H. and Bukau B. (1996) Escherichia coli trigger factor is a prolyl isomerase that associates with nascent polypeptide chains. Proc. Natl. Acad. Sci. USA 93: 4437-4441

34 Pfund C., Lopez-Hoyo N., Ziegelhoffer T., Schilke B. A., Lopez-Buesa P., Walter W. A. et al. (1998) The molecular chaperone Ssb from Saccharomyces cerevisiae is a component of the ribosome-nascent chain complex. EMBO J. 17: 3981-3989

35 Stoller G., Ruecknagel K. P., Nierhaus K. H., Schmid F. X., Fischer G. and Rahfeld J.-U. (1995) A ribosome-associated peptidyl-prolyl cis/trans isomerase identified as the trigger factor. EMBO J. 14: 4939-4948

36 Wiedmann B., Sakai H., Davis T. A. and Wiedmann M. (1994) A protein complex required for signal-sequence-specific sorting and translocation. Nature 370: $434-440$

37 Gautschi M., Lilie H., Fünfschilling U., Mun A., Ross S., Lithgow T. et al. (2001) RAC, a stable ribosome-associated complex in yeast formed by the DnaK-DnaJ homologs Sszlp and zuotin. Proc. Natl. Acad. Sci. USA 98: 3762-3767

38 Gautschi M., Mun A., Ross S. and Rospert S. (2002) A functional chaperone triad on the yeast ribosome. Proc. Natl. Acad. Sci. USA 99: 4209-4214

39 Yan W., Schilke B., Pfund C., Walter W., Kim S. and Craig E. A. (1998) Zuotin, a ribosome-associated DnaJ molecular chaperone. EMBO J. 17: 4809-4817
40 Hundley H., Eisenman H., Walter W., Evans T., Hotokezaka Y., Wiedmann M. et al. (2002) The in vivo function of the ribosome-associated Hsp70, Ssz1, does not require its putative peptide-binding domain. Proc. Natl. Acad. Sci. USA 99: 4203-4208

41 Jones G. W., Song Y. and Masison D. C. (2003) Deletion of the Hsp70 chaperone gene $S S B$ causes hypersensitivity to guanidine toxicity and curing of the [PSI+] prion by increasing guanidine uptake in yeast. Mol. Genet. Genomics 269: 304-311

42 Kim S. Y. and Craig E. A. (2005) Broad sensitivity of Saccharomyces cerevisiae lacking ribosome-associated chaperone Ssb or Zuo1 to cations, including aminoglycosides. Eukaryot. Cell 4: 82-89

43 Huang P., Gautschi M., Walter W., Rospert S. and Craig E. . (2005) The Hsp70 Ssz1 modulates the function of the ribosomeassociated J-protein Zuo1. Nat. Struct. Mol. Biol. 12: 497-504

44 Lopez N., Halladay J., Walter W. and Craig E. A. (1999) SSB, encoding a ribosome-associated chaperone, is coordinately regulated with ribosomal protein genes. J. Bacteriol. 181: 3136-3143

45 Rauch T., Hundley H. A., Pfund C., Wegrzyn R. D., Walter W., Kramer G. et al. (2005) Dissecting functional similarities of ribosome-associated chaperones from Saccharomyces cerevisiae and Escherichia coli. Mol. Microbiol. 57: 357-365

46 Ito K. (2005) Ribosome-based protein folding systems are structurally divergent but functionally universal across biological kingdoms. Mol. Microbiol. 57: 313-317

47 Hundley H. A., Walter W., Bairstow S. and Craig E. A. (2005) Human Mpp11 J protein: ribosome-tethered molecular chaperones are ubiquitous. Science 308: 1032-1034

48 Otto H., Conz C., Maier P., Wölfle T., Suzuki C. K., Jenö P. et al. (2005) The chaperones MPP11 and Hsp70L1 form the mammalian ribosome-associated complex (mRAC). Proc. Natl. Acad. Sci. USA 102: 10064-10069

49 Hansen W. J., Lingappa V. R. and Welch W. (1994) Complex environment of nascent polypeptide chains. J. Biol. Chem. 169: 26610-26613

50 Wiedmann B., Sakai H., Davis T. A. and Wiedmann M. (1994) A protein complex required for signal-sequence-specific sorting and translocation. Nature 370: 434-440

51 Fünfschilling U. and Rospert S. (1999) Nascent polypeptideassociated complex stimulates protein import into yeast mitochondria. Mol. Biol. Cell 10: 3289-3299

52 Rospert S., Dubaquie Y. and Gautschi M. (2002) Nascentpolypeptide-associated complex. Cell. Mol. Life Sci. 59: 1632-1639

53 Spreter T., Pech M. and Beatrix B. (2005) The crystal structure of archaeal nascent polypeptide-associated complex (NAC) reveals a unique fold and the presence of a ubiquitin-associated domain. J. Biol. Chem. 280: 15849-15854

54 Makarova K. S., Aravind L., Galperin M. Y., Grishin N. V., Tatusov R. L., Wolf Y. I. et al. (1999) Comparative genomics of the Archaea (Euryarchaeota): evolution of conserved protein families, the stable core and the variable shell. Genome Res. 9: 608-628

55 Reimann B., Bradsher J., Franke J., Hartmann E., Wiedmann M., Prehn S. et al. (1999) Initial characterization of the nascent polypeptide-associated complex in yeast. Yeast 15: 397-407

56 Lauring B., Sakai H., Kreibich G. and Wiedmann M. (1995) Nascent polypeptide-associated complex protein prevents mistargeting of nascent chains to the endoplasmic reticulum. Proc. Natl. Acad. Sci. USA 92: 5411-5415

57 Moller I., Beatrix B., Kreibich G., Sakai H., Lauring B. and Wiedmann M. (1998) Unregulated exposure of the ribosomal Msite caused by NAC depletion results in delivery of non-secretory polypeptides to the Sec61 complex. FEBS Lett. 441: 1-5

58 Bloss T. A., Witze E. S. and Rothman J. H. (2003) Suppression of CED-3-independent apoptosis by mitochondrial betaNAC in Caenorhabditis elegans. Nature 424: 1066-1071

59 Deng J. M. and Behringer R. R. (1995) An insertional mutation in the BTF3 transcription factor gene leads to an early postimplantation lethality in mice. Transgenic Res. 4: 264-269 
60 Markesich D. C., Gajewski K. M., Nazimiec M. E. and Beckingham K. (2000) bicaudal encodes the Drosophila beta NAC homolog, a component of the ribosomal translational machinery. Development 127: 559-572

61 Franke J., Reimann B., Hartmann E., Kohlerl M. and Wiedmann B. (2001) Evidence for a nuclear passage of nascent polypeptide-associated complex subunits in yeast. J. Cell Sci. 114: 2641-2648

62 Hofmann K. and Bucher P. (1996) The UBA domain: a sequence motif present in multiple enzyme classes of the ubiquitination pathway. Trends Biochem. Sci. 21: 172-173

63 Buchberger A. (2002) From UBA to UBX: new words in the ubiquitin vocabulary. Trends Cell Biol. 12: 216-221

64 Turner G. C. and Varshavsky A. (2000) Detecting and measuring cotranslational protein degradation in vivo. Science 289: $2117-2120$

65 Sato S., Ward C. L. and Kopito R. R. (1998) Cotranslational ubiquitination of cystic fibrosis transmembrane conductance regulator in vitro. J. Biol. Chem. 273: 7189-7192

66 Deuerling E., Schulze-Specking A., Tomoyasu T., Mogk A. and Bukau B. (1999). Trigger factor and DnaK cooperate in folding of newly synthesized proteins. Nature, 400: 693-696

67 Teter S. A., Houry W. A., Ang D., Tradler T., Rockabrand D., Fischer G. et al. (1999) Polypeptide flux through bacterial Hsp70: DnaK cooperates with Trigger Factor in chaperoning nascent chains. Cell 97: 755-765

68 Deuerling E., Patzelt H., Vorderwülbecke S., Rauch T., Kramer G., Schaffitzel E. et al. (2003) Trigger Factor and DnaK possess overlapping substrate pools and binding specificities. Mol. Microbiol. 47: 1317-1328

69 Ullers R. S., Luirink J., Harms N., Schwager F., Georgopoulos C. and Genevaux P. (2004) SecB is a bona fide generalized chaperone in Escherichia coli. Proc. Natl. Acad. Sci. USA 101: $7583-7588$

70 Vorderwulbecke S., Kramer G., Merz F., Kurz T. A., Rauch T., Zachmann-Brand B. et al. (2004) Low temperature or GroEL/ ES overproduction permits growth of Escherichia coli cells lacking trigger factor and DnaK. FEBS Lett. 559: 181-187

71 Genevaux P., Keppel F., Schwager F., Langendijk-Genevaux P. S., Hartl F. U. and Georgopoulos C. (2004) In vivo analysis of the overlapping functions of DnaK and trigger factor. EMBO Rep. 5: $195-200$

72 Patzelt H., Rudiger S., Brehmer D., Kramer G., Vorderwulbecke S., Schaffitzel E. et al. (2001) Binding specificity of Escherichia coli trigger factor. Proc. Natl. Acad. Sci. USA 98: 14244-14249

73 Rüdiger S., Germeroth L., Schneider-Mergener J. and Bukau B. (1997) Substrate specificity of the DnaK chaperone determined by screening cellulose-bound peptide libraries. EMBO J. 16: $1501-1507$

74 Zhu X., Zhao X., Burkholder W. F., Gragerov A., Ogata C. M., Gottesman M. et al. (1996) Structural analysis of substrate binding by the molecular chaperone DnaK. Science 272: 16061614

75 Rüdiger S., Buchberger A. and Bukau B. (1997) Interaction of Hsp70 chaperones with substrates. Nat. Struct. Biol. 4: 342-349

76 Hesterkamp T., Deuerling E. and Bukau B. (1997) The aminoterminal 118 amino acids of Escherichia coli trigger factor constitute a domain that is necessary and sufficient for binding to ribosomes. J. Biol. Chem. 272: 21865-21871

77 Zarnt T., Tradler T., Stoller G., Scholz C., Schmid F. X. and Fischer G. (1997) Modular structure of the Trigger Factor required for high activity in protein folding. J. Mol. Biol. 271: $827-837$

78 Kramer G., Rutkowska A., Wegrzyn R. D., Patzelt H., Kurz T. A., Merz F. et al. (2004) Functional dissection of Escherichia coli Trigger Factor: unraveling the function of individual domains. J. Bacteriol. 186: 3777-3784
79 Kramer G., Patzelt H., Rauch T., Kurz T. A., Vorderwulbecke S., Bukau B. et al. (2004) Trigger factor peptidyl-prolyl cis/trans isomerase activity is not essential for the folding of cytosolic proteins in Escherichia coli. J. Biol. Chem. 279: 14165-14170

80 Hesterkamp T. and Bukau B. (1996) Identification of the prolyl isomerase domain of Escherichia coli trigger factor. FEBS Lett. 385: $67-71$

81 Ferbitz L., Maier T., Patzelt H., Bukau B., Deuerling E. and Ban N. (2004) Trigger factor in complex with the ribosome forms a molecular cradle for nascent proteins. Nature 431: 590-596

82 Ludlam A. V., Moore B. A. and Xu Z. (2004) The crystal structure of ribosomal chaperone trigger factor from Vibrio cholerae. Proc. Natl. Acad. Sci. USA 101: 13436-13441

83 Kristensen O. and Gajhede M. (2003) Chaperone binding at the ribosomal exit tunnel. Structure 11: 1547-1556

84 Vogtherr M., Jacobs D. M., Parac T. N., Maurer M., Pahl A., Saxena K. et al. (2002) NMR solution structure and dynamics of the peptidyl-prolyl cis-trans isomerase domain of the trigger factor from Mycoplasma genitalium compared to FK506-binding protein. J. Mol. Biol. 318: 1097-1115

85 Hesterkamp T., Hauser S., Lütcke H. and Bukau B. (1996) Escherichia coli trigger factor is a prolyl isomerase that associates with nascent polypeptide chains. Proc. Natl. Acad. Sci. USA 93: 4437-4441

86 Valent Q. A., Kendall D. A., High S., Kusters R., Oudega B. and Luirink J. (1995) Early events in preprotein recognition in $E$. coli: interaction of SRP and trigger factor with nascent polypeptides. EMBO J. 14: 5494-5505

87 Mogk A., Mayer M. P. and Deuerling E. (2002) Mechanisms of Protein Folding: Molecular Chaperones and their Application in Biotechnology. Chembiochem. 3: 807-814

88 Houry W. A., Frishman D., Eckerskorn C., Lottspeich F. and Hartl F.U. (1999) Identification of in vivo substrates of the chaperonin GroEL. Nature 402: 147-154

89 Ewalt K. L., Hendrick J. P., Houry W. A. and Hartl F. U. (1997) In vivo observation of polypeptide flux through the bacterial chaperonin system. Cell 90: 491-500

90 Ying B. W., Taguchi H., Kondo M. and Ueda T. (2005) Cotranslational involvement of the chaperonin GroEL in the folding of newly translated polypeptides. J. Biol. Chem. 280: 12035-12040

91 Buchberger A., Schröder H., Hesterkamp T., Schönfeld H.-J. and Bukau B. (1996) Substrate shuttling between the DnaK and GroEL systems indicates a chaperone network promoting protein folding. J. Mol. Biol. 261: 328-333

92 Etchells S. A., Meyer A. S., Yam A. Y., Roobol A., Miao Y., Shao Y. et al. (2005) The cotranslational contacts between ribosome-bound nascent polypeptides and the subunits of the hetero-oligomeric chaperonin TRiC probed by photocrosslinking. J. Biol. Chem. 280: 28118-28126

93 McCallum C. D., Do H., Johnson A. E. and Frydman J. (2000) The interaction of the chaperonin tailless complex polypeptide 1 (TCP1) ring complex (TRiC) with ribosome-bound nascent chains examined using photo-cross-linking. J. Cell Biol. 149: 591-602

94 Frydman J., Nimmesgern E., Ohtsuka K. and Hartl F. U. (1994) Folding of nascent polypeptide chains in a high molecular mass assembly with molecular chaperones. Nature 370: 111-117

95 Horton L. E., James P., Craig E. A. and Hensold J. O. (2001) The yeast hsp70 homologue Ssa is required for translation and interacts with Sis1 and Pab1 on translating ribosomes. J. Biol. Chem. 276: $14426-14433$

96 Geissler S., Siegers K. and Schiebel E. (1998) A novel protein complex promoting formation of functional $\alpha$ - and $\gamma$-tubulin. EMBO J. 17: 952-966

97 Vainberg I. E., Lewis S. A., Rommelaere H., Ampe C. Vandekerckhove J., Klein H. L. et al. (1998) Prefoldin, a chaperone that delivers unfolded proteins to cytosolic chaperonin. Cell 93: 863-873 